\title{
MWP-fit: a program for multiple whole-profile fitting of diffraction peak profiles by ab initio theoretical functions
}

\author{
G. Ribárik, T. Ungár and J. Gubicza
}

Copyright (C) International Union of Crystallography

Author(s) of this paper may load this reprint on their own web site provided that this cover page is retained. Republication of this article or its storage in electronic databases or the like is not permitted without prior permission in writing from the IUCr. 
Journal of

\section{Applied Crystallography}

ISSN 0021-8898

Received 14 March 2001

Accepted 9 July 2001

\section{MWP-fit: a program for multiple whole-profile fitting of diffraction peak profiles by ab initio theoretical functions}

\author{
G. Ribárik, T. Ungár* and J. Gubicza \\ Department of General Physics, Eötvös University Budapest, H-1518, POB 32, Budapest, Hungary. \\ Correspondence e-mail: ungar@ludens.elte.hu
}

(C) 2001 International Union of Crystallography Printed in Great Britain - all rights reserved

\begin{abstract}
A computer program has been developed for the determination of microstructural parameters from diffraction profiles of materials with cubic or hexagonal crystal lattices. The measured profiles or their Fourier transforms are fitted by $a b$ initio theoretical functions for size and strain broadening. In the calculation of the theoretical functions, it is assumed that the crystallites have log-normal size distribution and that the strain is caused by dislocations. Strain and size anisotropy are taken into account by the dislocation contrast factors and the ellipticity of the crystallites. The fitting procedure provides the median and the variance of the size distribution and the ellipticity of the crystallites, and the density and arrangement of the dislocations. The efficiency of the program is illustrated by examples of severely deformed copper and ball-milled lead sulfide specimens.
\end{abstract}

\section{Introduction}

Diffraction peak profile analysis has been in general use for the investigation of the microstructure of crystalline materials since the 1920s (Becker, 1927). The original technique involved the measurement of the intensity distribution of Debye-Scherrer lines by photodensitometers, then progressed in the 1940s with the use of parafocusing powder diffractometers equipped with counters (Parrish, 1962), developing still further with the advent of high-resolution diffractometers in the home laboratory (Guinier, 1963; Wilkens \& Eckert, 1964), especially modern powder diffractometers using monochromatic radiation (Louër \& Langford, 1988), and high-resolution powder diffractometers at synchrotrons (Pattison et al., 2000). At the outset, the angular resolution was about $0.2^{\circ}$ (in $2 \theta$ ); it improved to about $0.1^{\circ}$ with the arrival of conventional powder diffractometers and is about $0.005^{\circ}$ with the high-resolution diffractometers. The obtainable peak to background ratio has improved to a similar extent, starting with about 20, improving for the $\theta-2 \theta$ diffractometers to 100 , or even somewhat better, and reaching $10^{3}$ to $10^{4}$ for the high-resolution diffractometers (Wilkens \& Eckert, 1964; Ungár et al., 1984; Pattison et al., 2000).

As for the peak shape, two fundamentally different approaches have been developed during the past four decades. In powder diffraction crystallography, the primary interest is in the crystal structure, the peak shape representing an auxiliary tool in the evaluation procedures (Rietveld, 1967, 1969; McCusker et al., 1999). In Rietveld structure refinement, the commonly used peak shapes are the following simple analytical functions: Gaussian, Lorentzian, pseudo-Voigtian, Voigtian, Pearson VII and empirical profile shape functions (McCusker et al., 1999). The Rietveld method has become a standard procedure in most powder diffraction applications, except the determination of microstructures, and is more or less completely satisfied by one or the other of the listed functions. There have been a few recent attempts to integrate microstructure determinations into the Rietveld method using these simple analytical functions
(Ungár, Leoni \& Scardi, 1999; Scardi \& Leoni, 1999; Langford et al., 2000).

From the point of view of peak profile analysis, the microstructure consists of two fundamentally different effects: (i) the effect of crystallite size and (ii) the effect of lattice distortions. The two have different diffraction-order dependences, which enables their separation (Wilson, 1958; Bertaut, 1950; Williamson \& Hall, 1953; Warren \& Averbach, 1952; Warren, 1959). Size broadening is caused by the finite column length of coherently scattering domains where this length is parallel to the diffraction vector (Guinier, 1963). Assuming the shape and the size distribution of the coherently scattering domains, the size profiles can be determined theoretically (Langford et al., 2000; Gubicza et al., 2000; Ungár, Gubicza et al., 2001). Lattice distortions are caused by lattice defects like dislocations, stacking faults, grain boundaries, inclusions, precipitates, etc. There have been many attempts to model strain broadening by describing lattice distortions as random displacements of atoms from their ideal crystal positions (Wilson, 1958, 1959; Stephens, 1999). It can be shown, however, that static random displacements are equivalent to thermal vibrations, which are well known to cause peak height reduction without peak broadening (Schwartz \& Cohen, 1977). It can also be shown that peak profile broadening in the vicinity of the fundamental Bragg reflections is most effectively caused by dislocations (Krivoglaz, 1969, 1996; Wilkens, 1970). Stacking faults, especially when they are of finite size, are also important lattice defects causing this type of peak broadening; however, most often they are outnumbered by the density of the dislocations. The physical reason for the relevance of dislocations in peak broadening is the $1 / r$ spatial dependence of their strain fields ( $r=$ distance from dislocations) (Krivoglaz, 1969). The displacement of atoms caused by dislocations is by far non-random. Krivoglaz \& Ryaboshapka (1963) and Wilkens (1970) have shown that there is a logarithmic singularity in the mean square strain of dislocations at small $L$ values ( $L$ is the Fourier transform variable). It can also be shown that the strain profiles are different from any of the 
simple analytical functions listed above (Wilkens, 1970). Highresolution X-ray diffraction experiments carried out on plastically deformed $\mathrm{Cu}$ and $\mathrm{Ni}$ single crystals have supported these results (Wilkens, 1988; Ungár et al., 1984; Hecker et al., 1997). From this we conclude that, though the simple analytical functions (see above) are a good approximation of diffraction peak profiles, especially for the purpose of structure refinement and qualitative or quantitative phase analyses, if the finer details of microstructure are required, neither the size nor the strain profiles can be approximated by simple analytical functions.

In a recent paper, a novel procedure was described to evaluate microstructural parameters of cubic or hexagonal crystalline materials obtained by the analysis of broadened diffraction peak profiles (Ungár, Gubicza et al., 2001). The Fourier coefficients of the measured physical profiles are fitted by Fourier coefficients of $a b$ initio physically well established functions of size and strain profiles (Ungár, Gubicza et al., 2001). The present paper describes the numerical procedures and the computer programs used in this evaluation method. The programs also enable the fitting of the measured physical intensity profiles by the inverse Fourier transform of $a b$ initio theoretical Fourier coefficients. The procedure has been tested by applying it to a nanocrystalline powder of galena $(\mathrm{PbS})$ and to a severely plastically deformed bulk copper specimen.

\section{Theoretical overview}

The Fourier coefficients of the peak profiles, $A(L)$, are the product of the size, $A^{S}$, and distortion, $A^{D}$, coefficients (Warren \& Averbach, 1952):

$$
A(L)=A^{S}(L) A^{D}(L)
$$

where $L$ is the variable of the Fourier transform. In the following, the theoretical Fourier transforms are even functions of $L$; therefore, the equations are given for $L \geq 0$.

\subsection{The distortion effect}

The distortion Fourier coefficients can be expressed in the following form (Warren \& Averbach, 1952):

$$
A^{D}(L)=\exp \left(-2 \pi^{2} g^{2} L^{2}\left\langle\varepsilon_{L}^{2}\right\rangle\right)
$$

where $g$ is the absolute value of the diffraction vector, $\left\langle\varepsilon_{L}^{2}\right\rangle$ is the mean square strain depending on the displacement of the atoms relative to their ideal positions, and the angle brackets indicate spatial averaging. Several authors have worked on the determination of the mean square strain, including Warren \& Averbach (1952), Krivoglaz \& Ryaboshapka (1963) and Wilkens (1970). Warren and co-workers (Warren \& Averbach, 1952; Warren, 1959) assumed either random atomic displacements and/or stacking faults. Krivoglaz (1969) and Wilkens (1970) assumed dislocations as the main source of peak broadening close to the fundamental Bragg positions, as mentioned before. Wilkens improved the model of Krivoglaz by introducing the effective outer cut-off radius of dislocations, $R_{e}^{*}$, instead of the crystal diameter. Assuming infinitely long parallel screw dislocations with a restrictedly random distribution, the mean square strain has been derived in the following closed form (Wilkens, 1970):

$$
\left\langle\varepsilon_{L}^{2}\right\rangle=(b / 2 \pi)^{2} \pi \rho C f\left(L / R_{e}^{*}\right),
$$

where $b$ is the absolute value of the Burgers vector, $\rho$ is the dislocation density, $C$ is the contrast factor of the dislocations and $f$ is the strain function (the Wilkens function). $f$ has the following explicit form (Wilkens, 1970, equations A6-A8 in Appendix A therein):

$$
\begin{aligned}
f^{*}(\eta)= & -\log \eta+\left(\frac{7}{4}-\log 2\right)+\frac{512}{90 \pi} \frac{1}{\eta} \\
& +\frac{2}{\pi}\left(1-\frac{1}{4 \eta^{2}}\right) \int_{0}^{\eta} \frac{\arcsin V}{V} \mathrm{~d} V \\
& -\frac{1}{\pi}\left(\frac{769}{180} \frac{1}{\eta}+\frac{41}{90} \eta+\frac{2}{90} \eta^{3}\right)\left(1-\eta^{2}\right)^{1 / 2} \\
& -\frac{1}{\pi}\left(\frac{11}{12} \frac{1}{\eta^{2}}+\frac{7}{2}+\frac{1}{3} \eta^{2}\right) \arcsin \eta+\frac{1}{6} \eta^{2}, \quad \text { if } \eta \leq 1, \\
f^{*}(\eta)= & \frac{512}{90 \pi} \frac{1}{\eta}-\left(\frac{11}{24}+\frac{1}{4} \log 2 \eta\right) \frac{1}{\eta^{2}}, \quad \text { if } \eta \geq 1,
\end{aligned}
$$

where $f\left(L / R_{e}^{*}\right)=f^{*}(\eta)$ and $\eta=(1 / 2) \exp (-1 / 4)\left(L / R_{e}^{*}\right)$. van Berkum et al. (1994) derived a simplified form of (4) by neglecting some terms. In the present work, we use the complete expression of the Wilkens function, especially since it has been shown experimentally that it describes well peak profiles of plastically deformed single crystals measured by high-resolution diffractometry (Wilkens \& Eckert, 1964). Note that in previous papers, $R_{e}=\exp (2) R_{e}^{*}=7.4 R_{e}^{*}$ was used as the effective outer cut-off radius of dislocations (Ungár et al., 1984; Wilkens, 1988; Hecker et al., 1997).

By inserting equation (3) into (2), the distortion Fourier transform is obtained:

$$
A^{D}(L)=\exp \left[-\left(\pi b^{2} / 2\right)\left(g^{2} C\right) \rho L^{2} f\left(L / R_{e}^{*}\right)\right] .
$$

Strain anisotropy is accounted for by the average contrast factors of the dislocations. For untextured polycrystals, the average contrast factors can be expressed by the fourth-order polynomials of the $h \mathrm{kl}$ indices (Ungár \& Tichy, 1999). For cubic crystals

$$
C=C_{h 00}\left(1-q H^{2}\right),
$$

where

$$
H^{2}=\frac{h^{2} k^{2}+h^{2} l^{2}+k^{2} l^{2}}{\left(h^{2}+k^{2}+l^{2}\right)^{2}} .
$$

For hexagonal crystals

$$
C=C_{h k 0}\left(1+a_{1} H_{1}^{2}+a_{2} H_{2}^{2}\right)
$$

where

$$
\begin{aligned}
& H_{1}^{2}=\frac{\left[h^{2}+k^{2}+(h+k)^{2}\right] l^{2}}{\left[h^{2}+k^{2}+(h+k)^{2}+(3 / 2)(a / c)^{2} l^{2}\right]^{2}}, \\
& H_{2}^{2}=\frac{l^{4}}{\left[h^{2}+k^{2}+(h+k)^{2}+(3 / 2)(a / c)^{2} l^{2}\right]^{2}},
\end{aligned}
$$

and $a / c$ is the ratio of the two lattice constants. Note that a formally similar equation to (6) has been derived by Stokes (1944) for a random displacement of atoms in elastically anisotropic cubic crystals. The constants $C_{h 00}$ and $C_{h k 0}$ are calculated on the basis of the crystallography of the dislocations and from the elastic constants of the crystal (see Ungár, Dragomir et al., 1999). The parameters $q, a_{1}$ and $a_{2}$ are the same for all reflections. One can see from equation (5) that the diffraction-order dependence of the distortion Fourier transform is given by $g^{2} C$. Note that for textured materials, the contrast factors should be calculated individually for each reflection (Borbély et al., 2000).

\subsection{The size effect}

According to previous works (Bertaut, 1950; Guinier, 1963), the Fourier transform of the intensity profile of the $h \mathrm{kl}$ diffraction peak 
equals the common volume of the crystal and its 'double' obtained by a translation $L$ in the direction normal to the reflecting lattice planes. For the calculation of the Fourier transform of the peak profile originating from a crystallite, let us divide the crystal into cylindrical columns normal to the lattice planes $h \mathrm{kl}$. Let us set $\mathrm{d} \sigma_{\mu}$ as the cross section of the columns, the heights of which in the crystal lie in the interval between $\mu$ and $\mu+\mathrm{d} \mu$. The common volume originating from these columns is (Guinier, 1963)

$$
A^{S}(L)=(1-|L| / \mu) \mu \mathrm{d} \sigma_{\mu} .
$$

The common volume of the irradiated crystallites and their 'double' shifted by $L$ can be obtained by summing for all columns existing in the crystallites. Assuming spherical crystallites and a log-normal crystallite size distribution, one can obtain

$$
A^{S}(L)=\int_{|L|}^{\infty}\left(1-\frac{|L|}{\mu}\right) \mu^{2}\left[\int_{\mu}^{\infty} f(x) \mathrm{d} x\right] \mathrm{d} \mu
$$

where $f(x)$ is the log-normal size distribution density function given by

$$
f(x)=\frac{1}{(2 \pi)^{1 / 2} \sigma} \frac{1}{x} \exp \left\{-\frac{[\log (x / m)]^{2}}{2 \sigma^{2}}\right\},
$$

where $\sigma$ is the variance and $m$ is the median of the distribution. The integral in equation (9) can be transformed into the following form:

$$
A^{S}(L)=\int_{|L|}^{\infty}\left(\mu^{2}-|L| \mu\right) \operatorname{erfc}\left[\frac{\log (\mu / m)}{2^{1 / 2} \sigma}\right] \mathrm{d} \mu,
$$

where erfc is the complementary error function defined as

$$
\operatorname{erfc}(x)=\frac{2}{\pi^{1 / 2}} \int_{x}^{\infty} \exp \left(-t^{2}\right) \mathrm{d} t
$$

Using substitutions and partial integration, the Fourier transform of the intensity profile arising from small distortion-free crystallites with spherical shape and log-normal size distribution can be written as

$$
\begin{aligned}
A^{S}(L)= & \frac{m^{3} \exp \left[(9 / 4)\left(2^{1 / 2} \sigma\right)^{2}\right]}{3} \operatorname{erfc}\left[\frac{\log (|L| / m)}{2^{1 / 2} \sigma}-\frac{3}{2} 2^{1 / 2} \sigma\right] \\
& -\frac{m^{2} \exp \left(2^{1 / 2} \sigma\right)^{2}}{2}|L| \operatorname{erfc}\left[\frac{\log (|L| / m)}{2^{1 / 2} \sigma}-2^{1 / 2} \sigma\right] \\
& +\frac{|L|^{3}}{6} \operatorname{erfc}\left[\frac{\log (|L| / m)}{2^{1 / 2} \sigma}\right] .
\end{aligned}
$$

The normalized Fourier transform can be obtained by dividing equation (13) by its maximal value at $L=0$ :

$$
A^{S}(0)=\frac{2 m^{3} \exp \left[(9 / 4)\left(2^{1 / 2} \sigma\right)^{2}\right]}{3} .
$$

Taking the inverse Fourier transform of equation (11), the size intensity profile has the following integral form (Gubicza et al., 2000):

$$
I^{S}(s)=\int_{0}^{\infty} \mu \frac{\sin ^{2}(\mu \pi s)}{(\pi s)^{2}} \operatorname{erfc}\left[\frac{\log (\mu / m)}{2^{1 / 2} \sigma}\right] \mathrm{d} \mu .
$$

Note that the size profile function for the same crystallite shape and size distribution has recently been derived by Langford et al. (2000). As a result of the different kind of derivation and summation, their formula [equation (21) of Langford et al., 2000] is different from equation (15) here; however, the two equations are mathematically equivalent.
Deriving (13) at $L=0$, we obtain the size parameter $L_{0}$, which equals the area-weighted mean column length:

$$
L_{0}=-\frac{A^{S}(0)}{\left[\mathrm{d} A^{S}(L) / \mathrm{d} L\right]_{L=0}}=\frac{2 m \exp \left[(5 / 4)\left(2^{1 / 2} \sigma\right)^{2}\right]}{3} .
$$

The maximal value of the size function (15) is

$$
I^{S}(0)=\int_{0}^{\infty} \mu^{3} \operatorname{erfc}\left[\frac{\log (\mu / m)}{2^{1 / 2} \sigma}\right] \mathrm{d} \mu .
$$

This integral can be determined by using similar transforms as we used in calculating (13). The volume-weighted mean column length is given as

$$
d=\frac{1}{\beta}=\frac{I^{S}(0)}{\int_{-\infty}^{\infty} I^{S}(s) \mathrm{d} s}=\frac{I^{S}(0)}{A^{S}(0)}=\frac{3 m \exp \left[(7 / 4)\left(2^{1 / 2} \sigma\right)^{2}\right]}{4} .
$$

Note that Hinds (1982) and Langford et al. (2000) obtained the same expressions for $d$ and $L_{0}$.

As can be seen from equation (13), the size Fourier transform for spherical crystallites is order independent. The anisotropy of the crystallite size is introduced by assuming that the crystallites each have the shape of an ellipsoid of revolution (Ungár et al., 2000). In this case, the Fourier transform of the size profile has the same form as equation (13), but the median of the size distribution depends on the indices of the reflection:

$$
m_{h k l}=m_{a} /\left[1+\left(1 / \varepsilon^{2}-1\right) \cos ^{2} \alpha\right]^{1 / 2},
$$

where $m_{a}$ is the median of the size distribution of the diameters of the ellipsoids perpendicular to the axis of revolution, $\varepsilon$ is the ratio of the diameters of the ellipsoids parallel and perpendicular to the axis of revolution (the so-called ellipticity), and $\alpha$ is the angle between the axis of revolution and the diffraction vector. If the relative orientations of the crystallographic directions with respect to the axis of revolution are known, $\cos \alpha$ can be expressed by the indices of the reflection. For example, if the axis of revolution is parallel to the edge of the elementary cell for cubic crystals or is perpendicular to the basal plane for hexagonal crystals, $\cos \alpha$ can be given as

$$
\cos \alpha=l /\left(h^{2}+k^{2}+l^{2}\right)^{1 / 2}
$$

or

$$
\cos \alpha=l /\left[(4 / 3)\left(c^{2} / a^{2}\right)\left(h^{2}+h k+k^{2}\right)+l^{2}\right]^{1 / 2},
$$

respectively.

\section{The method of multiple whole-profile (MWP) fitting}

The theoretical Fourier transform [given by equations (1), (4), (5), (6), (7), (13) and (14)] and the theoretical intensity function [given as the inverse Fourier transform of (1)] describe the shapes and the widths of the profiles, but do not provide the physically relevant heights of the profiles. Therefore, the measured intensity profiles and their Fourier transforms, as well as the fitting theoretical functions, are normalized by their maximum values in the fitting procedures, as follows.

(i) Multiple whole-profile fitting of the Fourier transforms. In this procedure, first the measured intensity profiles are Fourier transformed and normalized. Then all of them are fitted simultaneously by the normalized theoretical Fourier transform:

$$
A(L)=\frac{A^{S}(L)}{A^{S}(0)} \exp \left[-\frac{\pi b^{2}}{2}\left(g^{2} C\right) \rho L^{2} f\left(\frac{L}{R_{e}^{*}}\right)\right],
$$


where $A^{S}(L)$ and $A^{S}(0)$ are given by equations (13) and (14), respectively.

(ii) Multiple whole-profile fitting of the intensity profiles. In this procedure, first the measured intensity profiles are normalized. Then all of them are fitted simultaneously by the normalized theoretical intensity function:

$$
I(s)=F_{c}(s) / F_{c}(0)
$$

where $F_{c}$ is the cosine Fourier transform of (22):

$$
F_{c}(s)=2 \int_{0}^{\infty} A(L) \cos (2 \pi L s) \mathrm{d} L
$$

In both cases, (i) and (ii), all profiles are fitted simultaneously using a nonlinear least-squares algorithm, in which the sum of squared residuals (SSR), defined for the Fourier coefficients or the intensities as

$$
\chi^{2}=\sum_{h k l} \sum_{i}\left[A_{m}\left(h k l, L_{i}\right)-A_{\mathrm{th}}\left(h k l, L_{i}\right)\right]^{2}
$$

or

$$
\chi^{2}=\sum_{h k l} \sum_{i}\left[I_{m}\left(h k l, s_{i}\right)-I_{\mathrm{th}}\left(h k l, s_{i}\right)\right]^{2}
$$

respectively, is minimized, where the subscripts ' $m$ ' and 'th' indicate 'measured' and 'theoretical', respectively. In the present procedure, the profiles are weighted equally. It should be noted that the profiles which correspond to the same $g$ value but of which the $h k l$ indices are not permutations of each other (e.g. for face-centred cubic materials, 333 and 511 reflections) should be omitted in the evaluation procedure, because they are the sum of two profiles with different contrast factors [see equations (6) and (7)]. The theoretical functions are determined by the microstructural parameters $m, \sigma, \rho, R_{e}^{*}$ and $q$ (or $a_{1}$ and $a_{2}$ ) and the $h k l$ indices. The values of the microstructural parameters are refined during the fitting procedure. In the case of the ellipsoidal size function [defined by equations (13), (14) and (19), and (20) or (21)], the value of the ellipticity parameter, $\varepsilon$, can also be refined. The other parameters, the lattice constants, $a$ (and $c$ ), the Burgers vector length, $b$, and the contrast factors $C_{h 00}$ or $C_{h k 0}$, are input parameters of the fitting procedure. If the dislocation structure and the elastic constants of the material are not known, the length of the Burgers vector and the contrast factors, $C_{h 00}$ or $C_{h k 0}$, have to be estimated and the values of the microstructural parameters, $\rho$ and $R_{e}^{*}$, become uncertain up to a scaling factor. For example, in cubic crystals, the fitting procedure provides primarily the product $b^{2} \rho C_{h 00}$ from which, in principle, $\rho$ can only be obtained if $C_{h 00}$ and $b$ are known. Note, however, that $C_{h 00}$ changes slowly with the elastic constants and the dislocation character; therefore, this type of uncertainty is not serious (Ungár, Dragomir et al., 1999).

\section{Description of the program}

The purpose of the program is to prepare and evaluate experimental diffraction data by the method of multiple whole-profile fitting. $M W P$-fit consists of two main interactive programs: Mkdat and Evaluate. The function of the program Mkdat is to correct the measured profiles for (i) instrumental effects, (ii) background and (iii) overlapping peaks. The microstructural evaluation is carried out by the program Evaluate.

\subsection{Software and hardware environment}

The program was developed and tested under the Linux, Solaris and Digital Unix operating systems. The core of the Evaluate program is a modified version of GNUPLOT written in C language (the original GNUPLOT package is available from http://www. gnuplot.org). The Evaluate program is an interactive front-end written in the zsh shell-script language (available from http:// www.zsh.org). The Mkdat program is also written in zsh. Both the programs, Evaluate and Mkdat, require some of the GNU utilities (available from http://www.gnu.org). Parallel computing using the libpthread library (useful on SMP systems) is also supported. The program is now in a highly optimized state with no considerable hardware requirements. The typical run-time of an evaluation of nine separate profiles supposing about 50 iteration steps is about half a minute on a Compaq XP1000 workstation equipped with an Alpha Ev6 500 CPU. The same evaluation takes about $5 \mathrm{~min}$ on a PC equipped with an AMD 486 DX4/150 CPU. The memory requirement of the programs is not significant.

\subsection{Algorithms}

The program uses an implementation of the nonlinear leastsquares Marquardt-Levenberg (M-L) method (Levenberg, 1944; Marquardt, 1963) for the fitting, which is a standard part of GNUPLOT. The theoretical functions are written in thread-safe C code; therefore, no user-defined GNUPLOT functions are needed for the fitting. The integral in (4) is approximated by expanding the integrand into a Taylor series. The Fourier transform of the measured intensity profiles is calculated by a simple trapezoidal quadrature formula, sufficient for the non-equidistantly sampled input data. The cosine Fourier transform in (24) is computed by a fast Fourier transform algorithm (Cooley \& Tukey, 1965), using the implementation of Ooura (1996).

\subsection{The programs}

The program Evaluate needs the diffraction profiles as input data in two-column file format. The first column should consist of the position values scaled according to $\Delta K=2\left(\sin \theta-\sin \theta_{0}\right) / \lambda$, where $\theta_{0}$ is the Bragg angle of the peak and $\lambda$ is the wavelength of $\mathrm{X}$-rays. The second column should consist of the intensity values normalized according to their maximum. The program Evaluate can also be used for fitting if only the Fourier transforms of the profiles are given. If the file format of the input data is different from that described above, it can be corrected by the program Mkdat.

4.3.1. The program Mkdat. The program Mkdat recognizes ASCII input data in a file containing either the equidistantly sampled intensity values in one column or having two columns with $2 \theta$ in the first and the intensity values in the second. The files can contain any number of Bragg reflections. The preparation consists of the separation of overlapping peaks and background subtraction, and instrumental correction of the profiles. The program consists of the following steps.

(a) The program searches for the peaks if the whole powder diffractogram is available and gives a list of the positions and intensity maxima of the diffraction peaks.

(b) The user is given the option to keep or skip any of the peaks or to assign $h k l$ indices to the corresponding peaks.

(c) The principle of the peak separation is as follows. After the nonlinear $2 \theta \rightarrow K$ transformation $(K=2 \sin \theta / \lambda)$, the program prompts for the type (see below) and approximate positions of the selected and the unwanted overlapping peaks. Only in this particular correction procedure are the peaks fitted by simple mathematical 
functions, the type of which can be selected by the user: pseudoVoigtian, Voigtian or Pearson VII. The physical background is approximated by a polynomial. The order of the polynomial must be specified by the user. The 'background' of the peak selected for correction is the sum of the physical background and the other overlapping unwanted peaks. The parameters of the 'background' and the selected peak (including the positions and intensity maxima of the peaks) are refined using the nonlinear M-L algorithm of the GNUPLOT program. After the fit converges, the fitted 'background' is subtracted from the measured data. The position of the selected peak is shifted to $K=0$ and the intensity is normalized. If the fit was sufficiently good, only the separated and normalized peak remains.

(d) If instrumental profiles are available, the program can correct the measured profiles for the instrumental effect using the method of complex deconvolution (Stokes, 1948). However, the deconvolution of the physical and instrumental profiles has to be carried out with special care, particularly when the breadth of the observed profiles is close to that of the instrumental profiles (Snyder et al., 1999).

(e) Finally, the program saves the separated and corrected profile in the file hkl. dat in a two-column format in which the first and second columns contain the $\Delta K$ and the intensity values, respectively. If the instrumental effect was taken into account, the Fourier transform (which is a result of the deconvolution) is also saved.

4.3.2. The program Evaluate. The function of the program Evaluate is to evaluate the previously prepared data in order to obtain the microstructural parameters using the method of multiple wholeprofile fitting. Although this is an interactive program, it has an option 'auto' to run in the background using the input files and the default settings of the program. The steps of the evaluation procedure are as follow.

(a) Selection of the crystal system. The possible selections are cubic or hexagonal.

(b) Setting the value of the input parameters. The program prompts for the value of the lattice constant(s), the absolute value of the Burgers vector and the contrast factors $C_{h 00}$ or $C_{h k 0}$.

(c) Selection of the size function. The possible selections are: no size effect, spherical size function (default) or ellipsoidal size function.

(d) Specification of the sampling of the Fourier transform of the measured data. The number of samples (default 256) and the upper limit of sampling [the default is five times the full width at half-maximum (FWHM) value of the Fourier spectrum] may be specified.

(e) Computation of the normalized Fourier transform of the measured data. This step is skipped if the Fourier transforms are available from the instrumental correction [see the description of the program Mkdat, $\S 4.3 .1$, step $(d)$ ] or from previous runs. The Fourier transforms are saved after the computation.

( $f$ ) Specification of the initial values of the parameters and the point of stopping. The initial values of the fitting parameters are always saved for subsequent runs. The point of stopping is the convergence criteria of the M-L algorithm: the fitting is stopped if the specified maximal number of iterations (default 5000) is reached or $\Delta \chi^{2} / \chi^{2}$ between two iteration steps is less than the specified limit (default $10^{-9}$ ).

( $g$ ) Selection of the method of fitting. The procedure of multiple whole-profile fitting can be carried out by using the Fourier transforms or the intensity profiles.

(h) Fitting. The measured profiles and the fitting theoretical functions are plotted side by side in order of $g$ and are replotted in each step of iteration, so that it is possible to trace continuously how the theoretical profiles approach the measured data. The figure is saved after the fit converges. A typical plot for fitting the Fourier transforms is shown in Fig. 1.

(i) Printing of solutions. In addition to the resulting parameters $m$, $\sigma, \varepsilon, \rho, R_{e}^{*}$ and $q$ (or $a_{1}$ and $a_{2}$ ), the dislocation arrangement parameter $M^{*}=R_{e}^{*} \rho^{1 / 2}$ (introduced by Wilkens) and the size parameters $D, d$ and $L_{0}$ are printed. The parameter $D$ is defined by $D=$ $0.9 /$ FWHM, where FWHM is the full width at half-maximum of the size profile. The other two size parameters are defined by equations (18) and (16).

(j) Preparation of figures. If the Fourier transforms were fitted, the program also plots the intensity functions. If the intensity functions were fitted, the program also plots the Fourier transforms.

(k) Saving the results and removal of temporary files.

\subsection{Availability}

The program Evaluate is available via a World Wide Web front-end (http://www.renyi.hu/mwp) for non-commercial scientific purposes. The program Mkdat is available from the authors upon request (email: mwp@renyi.hu). The Web front-end is an HTML/CGI interface to the program Evaluate. All options listed in $\$ 4.3 .2$ are accessible. The main functions of the front-end are as follows.

(i) Uploading of the profile files using the POST method. The FILE Input must be implemented in the browser. Note that the newer browsers, like Netscape/Mozilla Navigator or Internet Explorer, are compatible.

(ii) Listing of the samples. The evaluation can be started by clicking on the name of the sample. The options selected in the previous run are loaded.

(iii) The evaluation of the profiles. After the fit terminates, the resulting microstructural parameters and the plots of the measured and theoretical profiles are presented.

(iv) Showing the results from previous runs. The resulting parameters in ASCII format and the plots of the measured and theoretical profiles in ASCII/PostScript/GIF format are preserved.

\subsection{Documentation}

This paper describes the purpose of the program, the theoretical aspects of the crystallographic problem, the method of solution and the miscellaneous functions of the program. For the documentation

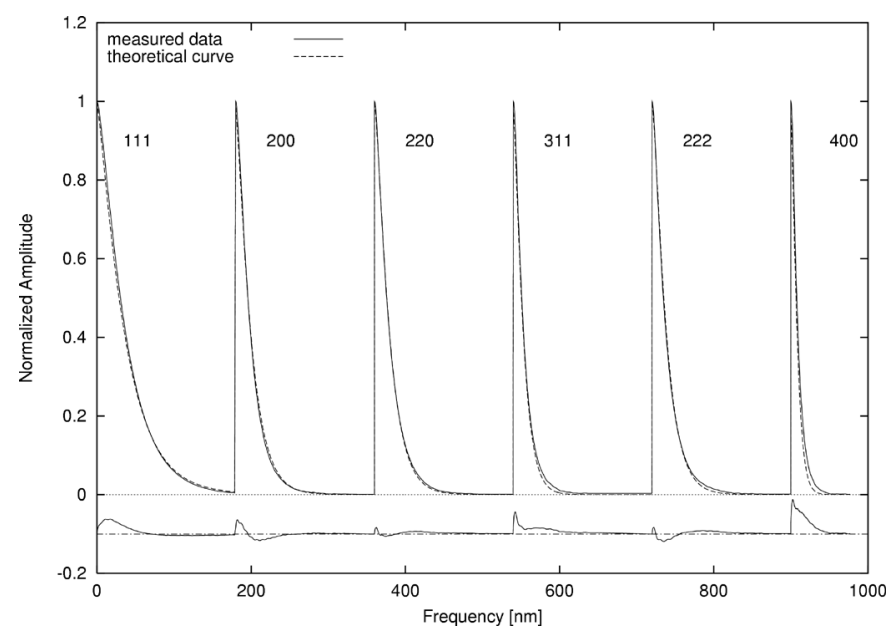

Figure 1

The measured (solid line) and theoretical fitted (dashed line) Fourier transforms for the copper sample as a function of the Fourier variable (frequency), $L$, plotted by the program Evaluate. The difference plot is also given, in the bottom of the figure. The indices of the reflections are indicated. 
about the Web front-end, visit http://www.renyi.hu/mwp/doc. A guide to the use and installation of the program Mkdat is distributed via email together with the program (see $\$ 4.4$ ).

\section{Examples of application}

Two representative examples of the application of the present procedure are presented: a submicrometre-grain-size copper specimen produced by severe plastic deformation (\$5.1) and a lead sulfide (galena) sample produced by ball milling (\$5.2).

\subsection{The microstructure of submicrometre-grain-size copper deter- mined by the MWP procedure}

High-purity oxygen-free copper that had been severely plastically deformed by a single pass of equal-channel angular pressing (ECA) was kindly provided by Professor Valiev (Valiev et al., 1994). The first six reflections were measured in a special high-resolution doublecrystal diffractometer with negligible instrumental broadening (Ungár \& Borbély, 1996). The MWP procedure was applied by fitting both the Fourier transforms and the intensity profiles. The measured and fitted Fourier coefficients and the measured and fitted intensity profiles are shown in Figs. 1 and 2, respectively. The good quality of the fitting can be observed for both the Fourier coefficients and the intensity functions. The intensity functions are shown in logarithmic scale, indicating that the fit is satisfactory down to $10^{-2}-10^{-3}$, depending on the quality of the measurement of the tail region of the profiles. The median, $m$, and the variance, $\sigma$, of the size distribution function, the three crystallite size parameters, $D, d$ and $L_{0}$, the $q$ parameter of the dislocation contrast factors, and the density, $\rho$, the outer cut-off radius, $R_{e}^{*}$, and the arrangement parameter, $M^{*}$, of the dislocations, obtained by fitting the Fourier transforms or the intensity profiles are listed in Table 1 . It can be seen that the size values obtained by fitting the intensity functions are smaller than the values given by the fitting of the Fourier transforms.

The crystallite size distribution has also been determined by transmission electron microscopy (TEM) (Ungár, Gubicza et al., 2001). The size distribution functions corresponding to the $m$ and $\sigma$ values in Table 1 and that obtained from the TEM measurements are shown in Fig. 3. It can be seen that the size distribution obtained from the Fourier coefficients shows a qualitatively better match with the

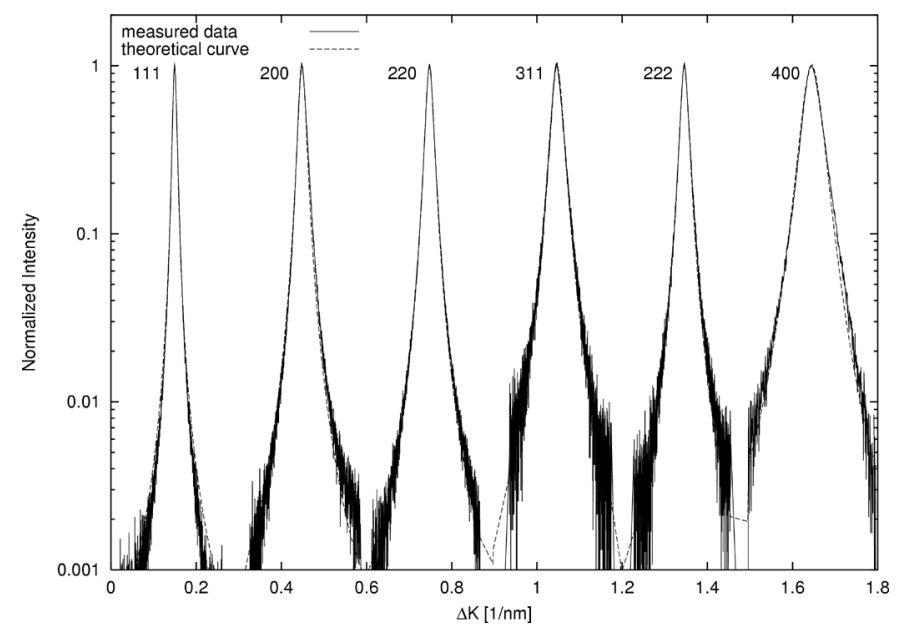

Figure 2

The measured (solid line) and theoretical fitted (dashed line) intensity profiles in logarithmic scale for the copper sample, plotted by the program Evaluate. The indices of the reflections are indicated.
Table 1

Data for the copper specimen.

(a) The median, $m$, and the variance, $\sigma$, of the size distribution function, and the three crystallite size parameters, $D, d$ and $L_{0}$, obtained by fitting the Fourier transforms or the intensity profiles.

\begin{tabular}{lccccr}
\hline & $m$ & $\sigma$ & $D$ & $d$ & $L_{0}$ \\
\hline MWP method using the Fourier transforms & 62 & 0.53 & 153 & 123 & 83 \\
MWP method using the intensity functions & 20 & 0.75 & 155 & 108 & 55 \\
\hline
\end{tabular}

(b) The $q$ parameter of the dislocation contrast factors, and the density, $\rho$, the outer cutoff radius, $R_{e}^{*}$, and the arrangement parameter, $M^{*}$, of the dislocations, obtained by fitting the Fourier transforms or the intensity profiles.

\begin{tabular}{lllll}
\hline & $q$ & $\rho\left(10^{14} \mathrm{~m}^{-2}\right)$ & $R_{e}^{*}(\mathrm{~nm})$ & $M^{*}$ \\
\hline MWP method using the Fourier transforms & 1.84 & 16.7 & 5.7 & 0.24 \\
MWP method using the intensity functions & 1.89 & 14.9 & 5.7 & 0.22 \\
\hline
\end{tabular}

TEM data than the size distribution function obtained from the fitting to the intensity functions. From this we conclude that if the tail regions of the profiles, in particular the intensity distributions below one half of the maximum, can be measured with sufficient accuracy, the fitting of the Fourier coefficients may provide microstructure data that are closer to reality. Note, however, that in the case of strongly overlapping peaks, fitting to the whole intensity of non-separated profiles would be most successful. This procedure, using the $a b$ initio physical functions, is still under development.

\subsection{The microstructure of ball-milled lead sulfide (galena) deter- mined by the MWP procedure}

A large number of natural $\mathrm{PbS}$ (galena) samples were ball-milled and in some cases heat treated in order to produce a microstructural map of galena produced by different crushing and heating procedures (Martinetto, 2000; Ungár, Martinetto et al., 2001). The purpose of this study was to investigate the manufacturing processes of ancient Egyptian cosmetics produced from galena (Walter et al., 1999; Martinetto, 2000).

Powder diffraction patterns of the crushed and heated natural galena specimens and of the archeological samples were measured at the high-resolution powder diffraction beamline BM16 of the ESRF

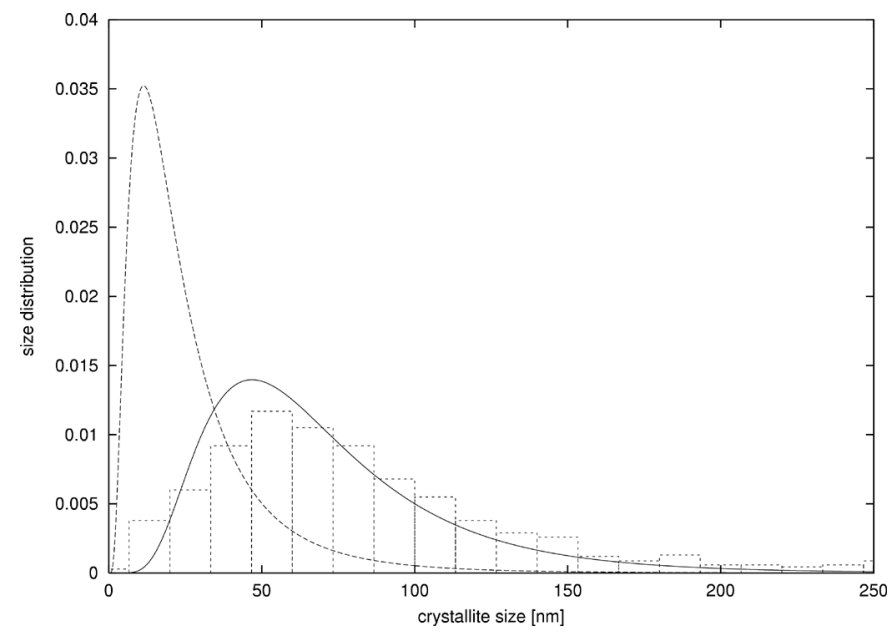

Figure 3

The crystallite size distribution density for the copper specimen obtained by fitting the Fourier transforms of the diffraction peaks (solid line) and the intensity profiles (dashed line), and that obtained from TEM micrographs (dotted columns). 
Table 2

Data for the PbS specimen.

(a) The median, $m$, and the variance, $\sigma$, of the size distribution function, and the three crystallite size parameters, $D, d$ and $L_{0}$, obtained by fitting the Fourier transforms or the intensity profiles.

\begin{tabular}{lrrrrr}
\hline & \multicolumn{1}{c}{ m } & \multicolumn{1}{c}{$\sigma$} & $D$ & $d$ & $L_{0}$ \\
\hline MWP method using the Fourier transforms & 21 & 0.73 & 148 & 100 & 52 \\
MWP method using the intensity functions & 8 & 0.87 & 140 & 85 & 35 \\
\hline
\end{tabular}

(b) The $q$ parameter of the dislocation contrast factors, and the density, $\rho$, the outer cutoff radius, $R_{e}^{*}$, and the arrangement parameter, $M^{*}$, of the dislocations, obtained by fitting the Fourier transforms or the intensity profiles.

\begin{tabular}{lllll}
\hline & $q$ & $\rho\left(10^{14} \mathrm{~m}^{-2}\right)$ & $R_{e}^{*}(\mathrm{~nm})$ & $M^{*}$ \\
\hline MWP method using the Fourier transforms & -6 & 13 & 3.2 & 0.12 \\
MWP method using the intensity functions & -4.9 & 12.5 & 2.8 & 0.1 \\
\hline
\end{tabular}

in Grenoble, France (Martinetto, 2000; Ungár, Martinetto et al., 2001). The powder diffractograms were evaluated to obtain the microstructural parameters by the procedure described here (Ungár, Martinetto et al., 2001). Figs. 4 and 5 show a representative example (the natural galena sample, planetary-ball-milled for $1 \mathrm{~h}$ ) of the fitting of the Fourier coefficients and the intensity profiles, respectively, by the program Evaluate. An excellent match between the measured and the fitted theoretical Fourier coefficients (Fig. 4) and between the measured and the fitted theoretical intensity functions (Fig. 5) is observed. The median, $m$, and the variance, $\sigma$, of the size distribution function, the three crystallite size parameters, $D, d$ and $L_{0}$, the $q$ parameter of the dislocation contrast factors, and the density, $\rho$, the outer cut-off radius, $R_{e}^{*}$, and the arrangement parameter, $M^{*}$, of the dislocations, obtained by fitting of the Fourier transforms or the intensity profiles are listed in Table 2.

\section{Problems occurring during the fitting procedure and how to avoid them}

After the evaluation of more than about 100 powder diffraction patterns by the present procedure, experience with the program has shown that in about $10 \%$ of the cases, the determination of $\rho$ and $R_{e}^{*}$ (or $M^{*}$ ) becomes unstable. This means that the M-L method enters

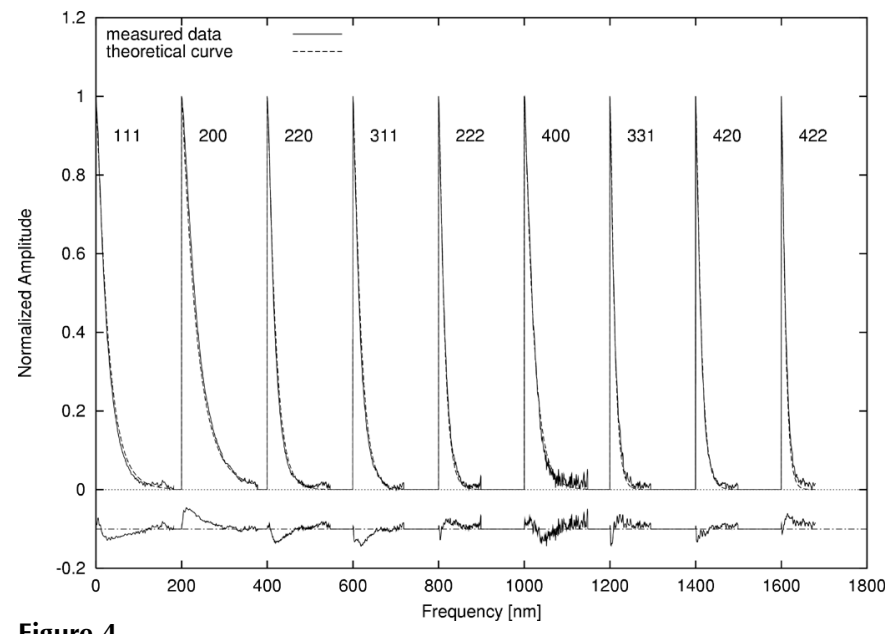

Figure 4

The measured (solid line) and theoretical fitted (dashed line) Fourier transforms for the PbS sample as a function of the Fourier variable (frequency), $L$, plotted by the program Evaluate. The difference plot is also given, in the bottom of the figure. The indices of the reflections are indicated. into an ill-defined asymptotic minimum of $\chi^{2}$ and the values of $\rho$ and $R_{e}^{*}$ tend to infinity or zero. The problem can be recognized by unrealistically high and low values of $\rho$ and $R_{e}^{*}$, and extremely large asymptotic standard errors given by the M-L code. The problem can be overcome by introducing a constraint by fixing the value of $M^{*}$. The application of this constraint means practically that the product of $\rho^{1 / 2}$ and $R_{e}^{*}$ is fixed. This constraint is an option of the program Evaluate, available when the initial parameter values are specified.

\section{Summary and conclusions}

A novel method and the corresponding software have been developed to provide the crystallite size and the crystallite size distribution function, the dislocation density and the dislocation character of crystalline materials from powder diffractograms. The procedure is based on $a b$ initio physical functions describing the concomitant size and strain contribution to broadened physical profiles. The size profile is evaluated by assuming spherical crystallite shape and lognormal size distribution functions. The Fourier transform of the size profile has been derived in a simple form that is easy to use in numerical calculations. Both the size profile and its Fourier transform have been derived for ellipsoidal crystallites also, allowing for anisotropy in the shape. Strain anisotropy is taken into account by assuming that strain is caused by dislocations. The spatial dependence of the mean square strain in the Fourier transform of the strain profiles is described by the function calculated for dislocations by Wilkens (1970). The $h k l$ dependence of the mean square strain is given by the dislocation contrast factors.

The software has been developed according to two different types of fitting philosophy. In the first, the Fourier coefficients of the measured physical profiles are fitted by the theoretical Fourier coefficients, consisting of the product of the theoretical size and strain Fourier coefficients. In the second, the measured physical profiles are fitted by the theoretical intensity profiles, produced by the inverse Fourier transform of the theoretical Fourier coefficients. In both cases, the microstructural parameters, $m, \sigma, \varepsilon, \rho, R_{e}^{*}$ and $q$ (or $a_{1}, a_{2}$ ), are provided by the nonlinear Marquardt-Levenberg fitting procedure. The software requires as input either the measured physical profiles after background subtraction and stripping of overlapping peaks, or the Fourier coefficients of the same measured physical

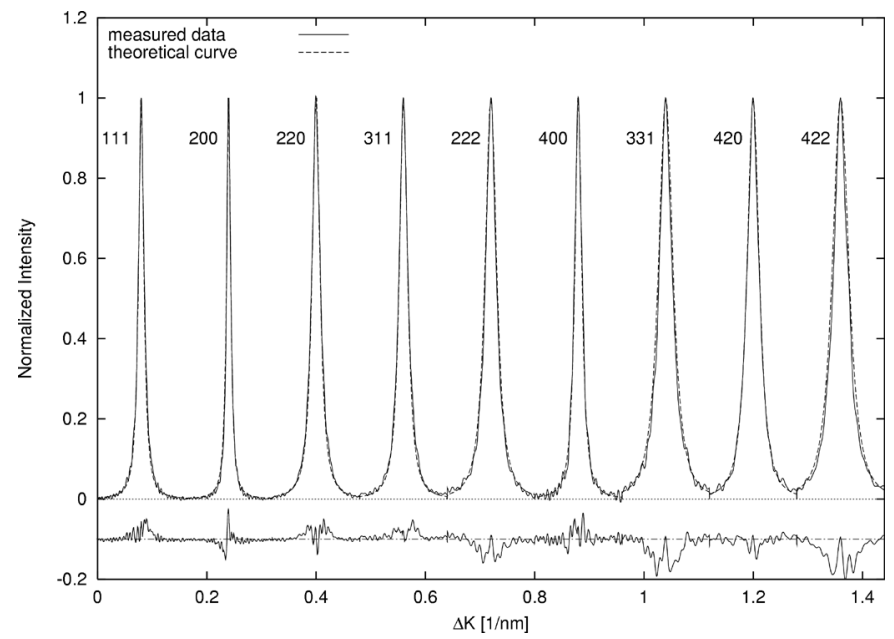

Figure 5

The measured (solid line) and theoretical fitted (dashed line) intensity profiles for the $\mathrm{PbS}$ sample, plotted by the program Evaluate. The difference plot is also given, in the bottom of the figure. The indices of the reflections are indicated. 
profiles after the same corrections. In the latter case, if instrumental correction was performed by the Stokes method, the input data would be provided directly.

The software is available via the Web front-end (http://www. renyi.hu/mwp) for users accepting the terms and conditions of usage. The program for separating overlapping peaks and background subtraction is available from the authors upon request.

The authors are indebted to Drs P. Martinetto and E. Dooryhee for the powder diffractogram of $\mathrm{PbS}$ (galena). The authors are grateful to the Alfréd Rényi Institute of Mathematics, Hungarian Academy of Sciences, for assuring the WWW front-end. The authors are grateful for the financial support of the Hungarian Scientific Research Fund, OTKA, grant Nos. T031786, T029701 and D29339.

\section{References}

Becker, K. (1927). Z. Phys. 42, 226-245.

Berkum, J. G. M. van, Vermuelen, A. C., Delhez, R., de Keijser, T. H. \& Mittemeijer, E. J. (1994). J. Appl. Cryst. 27, 345-357.

Bertaut, E. F. (1950). Acta Cryst. 3, 14-18.

Borbély, A., Driver, J. H. \& Ungár, T. (2000). Acta Mater. 48, 2005-2016.

Cooley, J. W. \& Tukey, J. W. (1965). Math. Comput. 19, 297-301.

Gubicza, J., Szépvölgyi, J., Mohai, I., Ribárik, G. \& Ungár, T. (2000). J. Mater. Sci. 35, 3711-3717.

Guinier, A. (1963). X-ray Diffraction. San Francisco: Freeman.

Hecker, M., Thiele, E. \& Holste, C. (1997). Mater. Sci. Eng. A, 234-236, 806809.

Hinds, W. C. (1982). Aerosol Technology: Properties, Behavior and Measurement of Airbone Particles. New York: Wiley.

Krivoglaz, M. A. (1969). Theory of X-ray and Thermal Neutron Scattering by Real Crystals. New York: Plenum.

Krivoglaz, M. A. (1996). X-ray and Neutron Diffraction in Nonideal Crystals. Berlin: Springer-Verlag.

Krivoglaz, M. A. \& Ryaboshapka, K. P. (1963). Fiz. Metall. 15, 18-31.

Langford, J. I., Louër, D. \& Scardi, P. (2000). J. Appl. Cryst. 33, 964-974.

Levenberg, K. (1944). Q. Appl. Math. 2, 164-168.

Louër, D. \& Langford, J. I. (1988). J. Appl. Cryst. 21, 430-437.

Marquardt, D. W. (1963). SIAM J. Appl. Math. 11, 431-441.

Martinetto, P. (2000). PhD thesis, Centre National de la Recherche Scientifique, Paris, France.
McCusker, L. B., Von Dreele, R. B., Cox, D. E., Louër, D. \& Scardi, P. (1999). J. Appl. Cryst. 32, 36-50.

Ooura, T. (1996). Miscellaneous program sources: http://momonga.t. u-tokyo.ac.jp/ ooura/

Parrish, W. (1962). Advances in X-ray Diffractometry and X-ray Spectrography. Eindhoven: Centrex.

Pattison, P., Knudsen, K. D. \& Fitch, A. N. (2000). J. Appl. Cryst. 33, 82-86.

Rietveld, H. M. (1967). Acta Cryst. 22, 151-152.

Rietveld, H. M. (1969). J. Appl. Cryst. 2, 65-71.

Scardi, P. \& Leoni, M. (1999). J. Appl. Cryst. 32, 671-682.

Schwartz, L. H. \& Cohen, J. B. (1977). Diffraction from Materials, pp. 360-371. Berlin: Springer-Verlag.

Snyder, R. L., Fiala, J. \& Bunge, H. J. (1999). Editors. Defect and Microstructure Analysis by Diffraction. IUCr/Oxford University Press.

Stephens, P. W. (1999). J. Appl. Cryst. 32, 281-289.

Stokes, A. R. \& Wilson A. J. C. (1944). Proc. Phys. Soc. London, 56, 174-183.

Stokes, A. R. (1948). Proc. Phys. Soc. London, 61, 382-393.

Ungár, T. \& Borbély, A. (1996). Appl. Phys. Lett. 69, 3173-3175.

Ungár, T., Dragomir, I., Révész, Â. \& Borbély, A. (1999). J. Appl. Cryst. 32, 992-1002.

Ungár, T., Gubicza, J., Ribárik, G. \& Borbély, A. (2001). J. Appl. Cryst. 34, 298-310.

Ungár, T., Gubicza, J., Ribárik, G. \& Zerda, T. W. (2000). Proceedings of the MRS Fall Meeting, Boston. In the press.

Ungár, T., Leoni, M. \& Scardi, P. (1999). J. Appl. Cryst. 32, 290-295.

Ungár, T., Martinetto, P., Ribárik, G., Dooryhée, E., Walter, P. \& Anne, M. (2001). In preparation.

Ungár, T., Mughrabi, H., Rönnpagel, D. \& Wilkens, M., (1984). Acta Metall. 32, 333-342.

Ungár, T. \& Tichy, G. (1999). Phys. Status Solidi A, 171, 425-434.

Valiev, R. Z., Kozlov, E. V., Ivanov, Yu. F., Lian, J., Nazarov, A. A. \& Baudelet, B. (1994). Acta Metall. Mater. 42, 2467-2476.

Walter, P., Martinetto, P., Tsoucaris, G., Bréniaux, R., Lefebvre, M. A., Richard, G., Talabot, J. \& Dooryhée, E. (1999). Nature (London), 397, 483483.

Warren, B. E. (1959). Prog. Met. Phys. 8, 147-202.

Warren, B. E. \& Averbach, B. L. (1952). J. Appl. Phys. 23, 497-497.

Wilkens, M. (1970). Fundamental Aspects of Dislocation Theory, Vol. II, edited by J. A. Simmons, R. de Wit \& R. Bullough, pp. 1195-1221, Natl Bur. Stand. (US) Spec. Publ. No. 317, Washington, DC, USA.

Wilkens, M. (1988). Proceedings of the 8th International Conference on the Strength of Metal Alloys (ICSMA 8), Tampere, Finland, 1988, edited by P. O. Kettunen, T. K. Lepistö \& M. E. Lehtonen, pp. 47-152. Oxford: Pergamon.

Wilkens, M. \& Eckert, H. (1964). Z. Naturforsch. Teil A, 19, 459-470.

Williamson, G. K. \& Hall, W. H. (1953). Acta Metall. 1, 22-31.

Wilson, A. J. C. (1958). Acta Cryst. 11, 227-228. 